\title{
VW HYI: A RAPIDLY COOLING WHITE DWARF?
}

\author{
BORIS T. GÄNSICKE, KLAUS BEUERMANN \\ Universitäts-Sternwarte, Geismarlandstr. 11, \\ 37083 Göttingen, FRG
}

\begin{abstract}
We estimate the post-outburst cooling time-scale of the white dwarf in VW Hyi, using the available quiescent IUE spectra. The determination of the white-dwarf temperature close to the outburst is hampered because disc emission still contributes to the SWP flux.
\end{abstract}

\section{Introduction}

Observations of the inter-outburst behavior of dwarf novae in the UV range are important in order to study the evolution of the disc/boundary layer as well as the possible cooling of the (accretion-heated) white dwarf. Alas, the white dwarf could be detected directly only in a small number of dwarf novae. The cooling of the white dwarf has been observed in U Gem (Long et al. 1994), WZ Sge (Sparks et al. 1993) and OY Car (Cheng et al. 1994). In the case of VW Hyi, Verbunt et al. 1987 detected a decline of the UV flux after an outburst, which has been interpreted by Meyer \& Meyer-Hofmeister (1994) by the evaporation of the inner (hot, UV-emitting) accretion disk. On the other hand, Sion et al. (1995) clearly identify the white dwarf in HST spectra.

\section{Analysis \& results}

We have retrieved all available IUE spectra of VW Hyi from the ULDA archive and selected $58 \mathrm{SWP} / \mathrm{LWR} / \mathrm{LWP}$ spectra obtained in quiescence. The UV flux shows an exponential decline, decreasing markedly slower after a superoutburst (Fig. 1). The individual spectra can be fitted reasonably well with $\log g=8$ white-dwarf model spectra by Ivan Hubeny, with the largest discrepancy in the LWP range. The radii derived for a distance of 65 pc (Fig. 1) are compatible with the assumed $0.6 \mathrm{M}_{\odot}$ white dwarf. Optical spectroscopy obtained in 1989 January shows the known orbital 


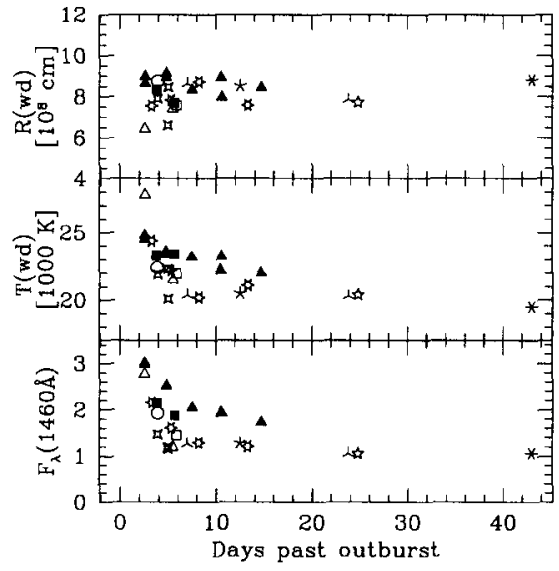

Figure 1: VW Hyi on the decline from the outburst. (a) UV flux in $10^{-13}$ ergs $\mathrm{cm}^{-2} \mathrm{~s}^{-1} \AA^{-1}$. Spectra taken after a normal outburst and after a superoutburst are indicated by open and filled symbols, respectively. (b) Effective temperatures derived from fitting whitedwarf models to the observed Ly $\alpha$ profile. (c) Radius of the emitting area at $d=65 \mathrm{pc}$.

modulation, presumably due to the varying aspect of the hot spot. Fitting the spot spectrum with Kurucz low-gravity models yields a temperature of $\sim 10000 \mathrm{~K}$, in agreement with the estimate of Mateo \& Szkody (1984). The contribution of the hot-spot in the LWP range is $\sim 10 \%$, consistent with the departure of the observed spectra from the white-dwarf models.

Taken at face value, the temperatures derived from fitting the observed Ly $\alpha$ profiles with white-dwarf models indicate that the white dwarf cools between two outbursts from $\sim 25000 \mathrm{~K}$ to $\sim 20000 \mathrm{~K}$. The cooling timescale seems to be of the same order as the duration of the preceding outburst, i.e. $\sim 5 \mathrm{~d}$ after a normal outburst and $\sim 15 \mathrm{~d}$ after a superoutburst. However, our ongoing analysis of the IUE spectra confirms the results by Huang et al. (1996) who show that an HST spectrum taken $10 \mathrm{~d}$ after a normal outburst contains a non-negligible contribution of a disc remnant. For the two spectra closest to the previous outburst, we find that the disc still contributes $\sim 60 \%$ (normal,$\triangle$ ) and $\sim 30 \%$ (super, $\Delta$ ) of the SWP flux, these spectra require a more detailed discussion. The spectra taken 24,25 and $43 \mathrm{~d}$ after the outburst show no further evolution and are compatible with a white dwarf of $T_{\mathrm{wd}} \simeq 20000 \mathrm{~K}$ without noticeable disc contribution.

\section{References}

Cheng, F.H., Marsh, T.R., Horne, K., Hubeny, I., 1994, AIP conf. proc. 308, 197

Huang, M., Sion, E.M., Hubeny, I., et al., these proceedings, p247

Long K.S., Sion, E.M., Huang, M., Szkody, P.,1994, Ap. J., 424, L49

Mateo, M., Szkody, P., 1984, AJ, 89, 863

Meyer, F., Meyer-Hofmeister, E., 1994, A\&A, 288, 175

Sion, E.M., Szkody, P., Cheng, F.H., Huang, M., 1995, Ap. J., 444, L97

Sparks, W.M., Sion, E.M., Starrfield, S.G., Austin, S., 1993, in "Cataclysmic Variables and Related Physics", eds O. Regev, G. Shaviv, Ann. Israel Phys. Soc., 10, 96

Verbunt, F., Hassall, B.J.M., Pringle, J.E. et al., 1987, MNRAS, 225, 113 